\title{
THE SELF-JOININGS OF RANK TWO MIXING TRANSFORMATIONS
}

\author{
DANIEL ULLMAN
}

(Communicated by R. Daniel Mauldin)

\begin{abstract}
The class of rank one mixing transformations has been known for some time to have certain so-called "exotic" properties. Specifically, any rank one mixing $T$ has only the two trivial factors, and nothing can commute with $T$ but powers of $T$ itself. This much was known to Ornstein in 1969 [3]. In 1983 J. King showed that rank one mixing transformations possess an even stronger property known as minimal self-joinings (MSJ). In this note we investigate how these results can be extended to the case of rank two mixing transformations. In this class, it is possible for there to exist nontrivial factors and commuting transformations: the square of a rank one mixing transformation and certain two point extensions of a rank one mixing transformation are rank two mixing [2]. What we prove is that, other than those two kinds of rank two mixing transformations, this class also has MSJ.
\end{abstract}

Definitions. Assume throughout that $T$ is a measure-preserving automorphism of a Lebesgue probability space $(X, \mathscr{B}, \mu)$. By a tower for $T$ of height $h$ we mean a set $B \in \mathscr{B}$ together with its first $h$ translates under $T$, provided that these translates are disjoint. $B$ is called the base of the tower $M=\bigcup_{i=0}^{h} T^{i} B$, and, for $0 \leq i \leq h, T^{i} B$ is called a level of the tower. (Notice that according to this terminology, a tower of height $h$ has $h+1$ levels.)

The well-known Rohklin Lemma asserts that, given any measure-preserving transformation $T$ and any $\varepsilon>0$, there is a tower $M$ for $T$ with $\mu M>1-\varepsilon$.

All partitions are presumed to consist of a finite number of measurable sets. If $\mathscr{P}_{j}$ is a sequence of partitions of $X$, we say $\mathscr{P}_{j}$ generates the $\sigma$-algebra $\mathscr{B}$ if $\mathscr{B}$ is the smallest $\sigma$-algebra containing all the $\mathscr{P}_{j}$.

A measure-preserving transformation $T$ is called rank $n$ or less if there is an infinite sequence of sets of $n$ disjoint towers $\left\{M_{j}(1), M_{j}(2), \ldots, M_{j}(n)\right\}_{j=1}^{\infty}$ such that the partitions $\mathscr{P}_{j}$ of $X$ given by

$$
\mathscr{P}_{j}=\bigcup_{i=1}^{n}\left\{\text { levels of } M_{j}(i)\right\} \cup\left\{X \backslash \bigcup_{i=1}^{n} M_{j}(i)\right\}
$$

generate the full $\sigma$-algebra $\mathscr{B}$. Given such a sequence, the towers with subscript $j$ are called $j$-towers. Obviously, we call a transformation rank $n$ (exactly) if it is rank $n$ or less but not rank $n-1$ or less.

Received by the editors November 16, 1989.

1980 Mathematics Subject Classification (1985 Revision). Primary 28D05. 
It turns out that the class of rank $n$ or less transformations can be characterized as the family of automorphisms isomorphic to a cutting and stacking transformation of the unit interval with $n$ or fewer towers at each stage. (The $n=1$ version of this fact was first prove by Baxter [1]; the case of general $n$ is not much harder.) I do not describe the details of such a construction nor refer to cutting and stacking in the sequel. Suffice it to say that this result allows us to assume that the partitions $\mathscr{P}_{j}$ in (1) form a nested sequence, each refining the previous.

A $T \times T$-invariant measure $\bar{\mu}$ on the Cartesian product space $X \times X$, which projects to $\mu$ in both directions, is called a self-joining of $T$. Any $T$ has certain obvious ergodic self-joinings; namely, product measure $\mu \times \mu$ and the so-called off-diagonal measures-measures defined on measurable rectangles by $\bar{\mu}(A \times B)=\mu\left(A \cap T^{-n} B\right)$ for some integer $n$. If these are the only ergodic selfjoinings, then $T$ is said to have minimal self-joinings (MSJ). This definition is due to D. Rudolph [4].

The commutant of $T$ is the group of measure-preserving transformations that commute with $T$ modulo the (necessarily normal) subgroup of integral powers of $T$. A factor $\sigma$-algebra is a $T$-invariant sub- $\sigma$-algebra of $\mathscr{B} . \mathscr{B}$ itself and $\{\phi, X\}$ are factors of any $T$ and are called trivial. It is not hard to see that MSJ implies that $T$ has trivial commutant and factors. For if $S$ commutes with $T$ and is not a power of $T, \bar{\mu}(A \times B)=\mu\left(A \cap S^{-n} B\right)$ defines a new ergodic self-joining, and if $\mathscr{F}$ is a nontrivial $T$-invariant (factor) $\sigma$ algebra, then $\bar{\mu}(A \times B)=\int_{X} E(A \mid \mathscr{F}) E(B \mid \mathscr{F}) d \mu$ defines a self-joining not all of whose ergodic components can be product measure or off-diagonal measures.

A map $T$ is mixing (or satisfies the mixing property) if, for all $A$ and $B \in \mathscr{B}, \mu\left(A \cap T^{-n} B\right) \rightarrow \mu A \mu B$ as $n$ goes to infinity.

\section{THE CLASSIFICATION THEOREM}

Theorem. If $T$ is a rank two mixing transformation, then either

(1) $T$ is the square of a rank one mixing transformation,

(2) $T$ is a two point extension of a rank one mixing transformation, or

(3) $T$ has MSJ.

The proof proceeds through a series of lemmas. Throughout, $T$ acts on a space $(X, \mathscr{B}, \mu)$. For each natural number $j, X$ is partitioned into two towers $M_{j}(1)$ and $M_{j}(2)$, called $j$-towers, and a set $X \backslash\left(M_{j}(1) \cup M_{j}(2)\right) . M_{j}(1)$ and $M_{j}(2)$ have bases $B_{j}(1)$ and $B_{j}(2)$ and heights $h_{j}(1)$ and $h_{j}(2)$, respectively. Levels of $j$-towers are naturally called $j$-levels. The partitions $\mathscr{P}_{j}$ of $X$ whose elements are $j$-levels successively refine each other and generate $\mathscr{B}$. We assume that the real numbers $\mu\left(M_{j}(1)\right)$ and $\mu\left(M_{j}(2)\right)$, for $j=1,2,3, \ldots$, are bounded away from zero, by $\gamma$, say. (Otherwise, $T$ is rank one.) Finally, we assume that the base of the $j$-towers are a union of at least twenty $(j+1)$ levels; this can be arranged by passing to a subsequence of towers $j_{k}$.

We begin with a lemma that guarantees that almost all points in $X$ lie in the middle $98 \%$ of their $j$-tower for at least $3 / 4$ of all $j$. For $0<\alpha<1 / 2$ and $i=1$ or 2 , let

$$
D_{j}(i, \alpha)=\bigcup_{k=\left\lfloor\alpha h_{j}(i)\right\rfloor}^{\left\lfloor(1-\alpha) h_{j}(i)\right\rfloor} T^{k} B_{j}(i)
$$


and set

$$
D_{j}(\alpha)=\bigcup_{i=1}^{2} D_{j}(i, \alpha)
$$

This is the set of points on one of the $j$-towers that are not too close to the top or bottom.

Lemma 1. Let

$$
S(\alpha)=\left\{x \in X \mid \liminf _{N \rightarrow \infty} \frac{\#\left\{j \mid x \in D_{j}(\alpha), 1 \leq j \leq N\right\}}{N} \geq 3 / 4\right\} .
$$

Then $\mu S(1 / 100)=1$.

Proof. First notice that $x \in S(1 / 99)$ implies that $T^{n} x \in S(1 / 100)$ for all integers $n$. Consequently, $T^{n} S(1 / 99) \subset S(1 / 100)$, so

$$
\bigcup_{n=-\infty}^{\infty} T^{n} S(1 / 99) \subset S(1 / 100)
$$

To show that $\mu S(1 / 100)=1$, it is enough to show that $\mu S(1 / 99)>0$, since then the left-hand side of (2) is an invariant set of positive measure.

The idea is that the sets $D_{j}(1 / 99)$, for $j=1,2,3, \ldots$, are close to being independent sets. We modify them slightly (to produce independent sets) and then use the law of large numbers.

Find four natural numbers $a(1), a(2), b(1)$, and $b(2)$ such that, if

$$
E_{j}=\bigcup_{i=1}^{2} \bigcup_{k=a(i)}^{b(i)} T^{k} B_{j}(i)
$$

then

$$
\begin{gathered}
E_{j} \subset D_{j}(1 / 99), \\
\mu E_{j} \geq 3 / 4,
\end{gathered}
$$

and

$$
\bigcup_{i=1}^{2} T^{a(i)} B_{j}(i) \subset \bigcup_{i=1}^{2} B_{j-1}(i), \quad \text { and } \bigcup_{i=1}^{2} T^{b(i)} B_{j}(i) \subset \bigcup_{i=1}^{2} T^{h_{j-1}(i)} B_{j-1}(i) .
$$

Condition (5) says simply that $E_{j}$ must be a union of $j$-levels the first of which is a subset of the base of a $(j-1)$-tower and the last of which is a subset of the top level of a $(j-1)$-tower. That $a(1), a(2), b(1)$, and $b(2)$ can be chosen satisfying (3) and (4) requires that the $j$-towers be many times higher than the $(j-1)$-towers, which is equivalent to the assumption made at the end of the first paragraph after the statement of the theorem. is,

Let $\nu$ be the measure $\mu$ restricted to $M_{1}(1) \cup M_{1}(2)$ and normalized; that

$$
\nu(B)=\frac{\nu\left(B \cap\left(M_{1}(1) \cup M_{1}(2)\right)\right)}{\mu\left(M_{1}(1) \cup M_{1}(2)\right)}
$$

for $B \subset X$. Then the sets $E_{j}$ are independent with respect to $\nu$, since no data of the form $x \in E_{k}$ or $x \notin E_{k}$ for $k>j$ give any information about which $j$-level the point $x$ is on. 
Hence the strong law of large numbers tells us that, for $\nu$-almost every $x$,

$$
\lim _{N \rightarrow \infty} \frac{\#\left\{j \mid x \in E_{j}, 1 \leq j \leq N\right\}}{N} \text { exists and is } \geq 3 / 4 \text {. }
$$

But $E_{j} \subset D_{j}(1 / 99)$ and so $\nu S(1 / 99)=1$. This implies that $\mu S(1 / 99)>0$ and so $\mu S(1 / 100)=1$ as required.

Hereafter, we assume that the property that Lemma 1 ascribes to almost all points in fact holds for all points. We may do this, since the property is invariant.

For any pair $(x, y) \in X \times X$, we set $r_{j}$ equal to the greatest nonpositive integer $i$ such that either $T^{i} x$ or $T^{i} y$ is in $B_{j}(1) \cup B_{j}(2)$. Similarly, let $s_{j}$ be the smallest nonnegative integer $i$ such that either $T^{i} x$ or $T^{i} y$ is in the top level of a $j$-tower, that is, in the set $\bigcup_{i=1}^{2} T^{h_{j}(i)} B_{j}(i)$. We say that the interval of integers between $r_{j}$ and $s_{j}$ is a frame (the $j$-frame) for $(x, y)$. Note that, when $j$ is sufficiently large, both $x$ and $y$ are on $j$-towers, and for such $j$, the $j$-frame is shorter than both $h_{j}(1)$ and $h_{j}(2)$.

Lemma 2. Suppose $\bar{\mu}$ is a self-joining of $T,(x, y) \in X \times X$, and $x$ and $y$ are not on the same orbit of $T$. Suppose also that there is a subsequence $J \subset N$ of natural numbers $j$ such that (1) $x$ and $y$ lie in the middle $98 \%$ of the same $j$-tower, and (2) for all sets $A$ and $B$ in $X$ that are unions of levels of some tower,

$$
\frac{\#\left\{k \mid T^{k} x \in A, T^{k} y \in B, \text { and } r_{j} \leq k \leq s_{j}\right\}}{s_{j}-r_{j}+1} \rightarrow \bar{\mu}(A \times B)
$$

as $j$ goes to infinity along the subsequence $J$. Then $\bar{\mu}=\mu \times \mu$, product measure. Comment. Condition (2) of the lemma is certainly satisfied if $(x, y)$ is generic for $\bar{\mu}$, which means that for any sets $A$ and $B$ made out of levels of some tower,

$$
\frac{\#\left\{k \mid T^{k} x \in A, T^{k} y \in B \text {, and }-m \leq k \leq n\right\}}{m+n+1} \rightarrow \bar{\mu}(A \times B) \quad \text { as } m, n \rightarrow \infty .
$$

Proof. For $j \in J$, let $F_{j}$ be the phase shift between the $j$-block in which $x$ sits and the $j$-block in which $y$ sits. By this I mean that if $n(x)$ and $n(y)$ are the smallest nonnegative integers such that $x \in T^{n(x)}\left(B_{j}(1) \cup B_{j}(2)\right)$ and $y \in T^{n(y)}\left(B_{j}(1) \cup B_{j}(2)\right)$, then $F_{j}=n(y)-n(x)$. Since $x$ and $y$ are assumed to be on different orbits of $T,\left|F_{j}\right| \rightarrow \infty$, as $j$ goes to infinity along $J$.

Let $A$ and $B$ be subsets of $X$ made up of $j_{0}$-levels. Fix $\varepsilon>0$. Because $T$ is mixing, there is an integer $N$ so that

$$
\left|\frac{\mu\left(A \cap T^{n} B\right)}{\mu A \mu B}-1\right|<\varepsilon \text { for all } n \text { with }|n|>N .
$$

Pick $j \in J$ so large that

$$
\begin{gathered}
j \geq j_{0}, \\
\left|F_{j}\right|>N,
\end{gathered}
$$

and

$$
\left|\frac{\#\left\{k \mid T^{k} X \in A, T^{k} y \in B, \text { and } r_{j} \leq k \leq s_{j}\right\}}{\left(s_{j}-r_{j}+1\right) \bar{\mu}(A \times B)}-1\right|<\varepsilon .
$$

By switching the roles of $x$ and $y$ if necessary, we may assume that $F_{j} \geq 0$, so that Figure 1 is qualitatively correct. 


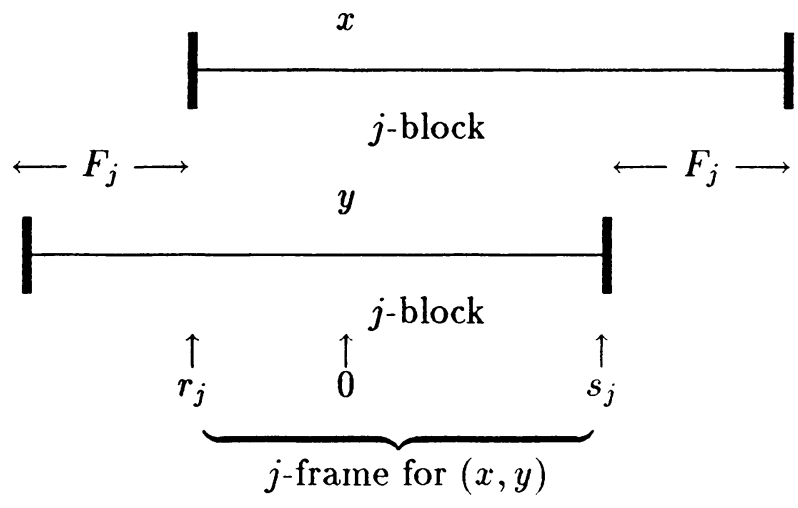

FIGURE 1

We now calculate:

$$
\begin{aligned}
\bar{\mu}(A \times B)(1 \pm \varepsilon) & =\frac{1}{s_{j}-r_{j}+1} \sum_{k=r_{j}}^{s_{j}} \chi_{A}\left(T^{k} x\right) \chi_{B}\left(T^{k} y\right) \\
& =\frac{1}{s_{j}-r_{j}+1} \sum_{k=r_{j}}^{s_{j}} \chi_{A}\left(T^{k} x\right) \chi_{B}\left(T^{k+F_{j}} x\right) \\
& =\frac{1}{s_{j}-r_{j}+1} \sum_{k=r_{j}}^{s_{j}} \chi_{A \cap T^{-F_{j}}}\left(T^{k} x\right) .
\end{aligned}
$$

The first equality here comes from (9) and the second holds because $\left(T^{k} x, T^{k} y\right)$ $\in A \times B$ if and only if $T^{k} x \in A$ and $T^{k+F_{j}} x \in B$, when $r_{j} \leq k \leq s_{j}$. If $x \in M_{j}(i)$, let

$$
C=\bigcup_{k=0}^{s_{j}-r_{j}} T^{k} B_{j}(i)
$$

the set of points on the same $j$-tower as $x$, at a height between 0 and $s_{j}-r_{j}$. Now (7) implies that $A \cap T^{-F_{j}} B \cap C$ is made entirely of complete $j$-levels, hence expression (10) above implies

$$
\bar{\mu}(A \times B)(1 \pm \varepsilon)=\mu\left(A \cap T^{-F_{J}} B \mid C\right) .
$$

Since $C$ is at least one hundreth of $M_{j}(i)$ (because of condition (1) of the lemma) we see that $\mu C>\gamma / 100$. (Remember that $\gamma=\inf _{i, j} \mu M_{j}(i)$.) Thus,

$$
\begin{aligned}
\mu\left(A \cap T^{-F_{j}} B \mid C\right) & =\mu\left(A \cap T^{-F_{j}} B \cap C\right) / \mu C \\
& \leq \mu\left(A \cap T^{-F_{j}} B\right) / \mu C<(100 / \gamma) \mu\left(A \cap T^{-F_{j}} B\right),
\end{aligned}
$$

which, owing to (6) and (8), is $\leq(100 / \gamma) \mu A \mu B(1 \pm \varepsilon)$. The outcome of this calculation is that

$$
\bar{\mu}(A \times B)(1-\varepsilon) \leq[100(1+\varepsilon) / \gamma] \mu A \mu B,
$$


or

$$
\bar{\mu}(A \times B) \leq L \mu A \mu B,
$$

where $L$ is a constant independent of $A$ and $B$. Since this inequality holds for all $A$ and $B$ made up of $j_{0}$-levels, and since $j_{0}$ is arbitrary,

$$
\bar{\mu}<<\mu \times \mu \text {. }
$$

Finally, since $\bar{\mu}$ is $T \times T$-invariant (and normalized), we conclude that $\bar{\mu}=\mu \times \mu$ and the lemma is proved.

Lemma 3. Suppose $\bar{\mu}$ is an ergodic self-joining of $T$ that is not product or offdiagonal measure. Then for all $\varepsilon>0$, there is a natural number $N$ so that $j<N$ implies

$$
\bar{\mu}\left(\bigcup_{i=1}^{2}\left(D_{j}(i, 1 / 100) \times D_{j}(i, 1 / 100)\right)\right)<\varepsilon .
$$

Proof. Suppose that there are an $\varepsilon>0$ and a sequence of sets $A_{j} \subset$ $\bigcup_{i=1}^{2}\left(D_{j}(i, 1 / 100) \times D_{j}(i, 1 / 100)\right)$ with $\bar{\mu} A_{j}>\varepsilon$, for infinitely many $j$. Then $\bar{\mu}\left(\liminf A_{j}\right)>\varepsilon$, so $\liminf _{j} A_{j}$ has a generic point $(x, y)$ for $\bar{\mu}$. If $x$ and $y$ were on the same orbit, then $\bar{\mu}$ would be an off-diagonal measure. If $x$ and $y$ were not on the same orbit, then we could infer from $(x, y) \in \liminf _{j} A_{j}$ that condition (1) of lemma (2) is satisfied. The genericity of $(x, y)$ gives us condition (2) of that lemma, which would imply that $\bar{\mu}$ is product measure. The lemma is proved.

Lemma 4. Suppose that $\bar{\mu}$ is an ergodic joining of $T$ that is not product measure. Assume that both $\left(x, y_{1}\right)$ and $\left(x, y_{2}\right)$ are generic for $\bar{\mu}$ and that $x$ and $y_{i}$ are on opposite $j$-towers for all sufficiently large $j$, for $i=1$ and 2 . Then $y_{1}=y_{2}$. Proof. $y_{1}$ and $y_{2}$ are on the same $j$-tower for all sufficiently large $j$. Let $r_{j}$ and $s_{j}$ be the beginning and the end of the $j$-frame for $\left(y_{1}, y_{2}\right)$.

Skip those $j$ for which either $y_{1}$ or $y_{2}$ is not in $D^{j}(1 / 100)$. Lemma 1 assures us that we are left with infinitely many $j$. Along the subsequence that remains, we have $s_{j}-r_{j}+1 \rightarrow \infty$.
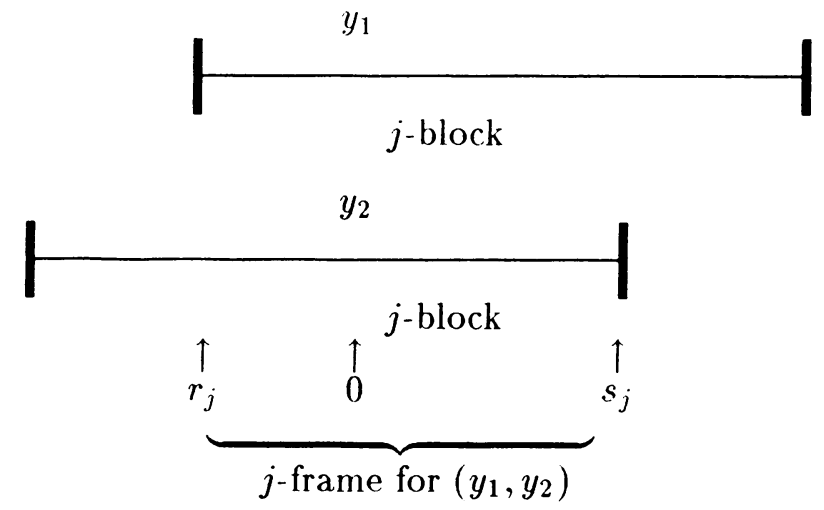

FIGURE 2 
There is a natural topology on $X$ associated with the towers $M_{j}(i)$, and that is the one generated by levels of towers. That is, a set is open (and closed) if it is a countable union of elements of the partitions $\mathscr{P}_{j}$. Let

$$
\bar{\nu}_{j}=\frac{1}{s_{j}-r_{j}+1} \sum_{k=r_{j}}^{s_{j}} \delta_{\left(T_{y_{1}}^{k}, T_{y_{2}}^{k}\right)},
$$

where $\delta_{(x, y)}$ is the probability measure putting all its mass on the point $(x, y)$. Set $\bar{\nu}$ equal to any weak * limit point of $\left\{\bar{\nu}_{j}\right\} . \bar{\nu}$ is a measure on $X \times X$, which projects to $\mu$ along both coordinates, since $y_{1}$ and $y_{2}$ are generic for $\mu$. Since the $\bar{\nu}_{j}$ become more and more $T \times T$-invariant as $j$ grows large, one can use a standard $\varepsilon / 3$ argument to prove that $\bar{\nu}$ is $T \times T$-invariant. This makes $\bar{\nu}$ a self-joining of $T$. Moreover, since the indicator function of any open set is continuous (the topology is totally disconnected), $\bar{\nu}$ satisfies

$$
\bar{\nu}_{j}(A \times B) \rightarrow \bar{\nu}(A \times B)
$$

for all sets $A$ and $B$ in $X$ that are made out of levels.

We now argue that $y_{1}$ and $y_{2}$ must be on the same orbit of $T$. If not, then $\bar{\nu}$ has been arranged to satisfy the conditions of Lemma 2, with $\left(y_{1}, y_{2}\right)$ in place of $(x, y)$. That lemma tells us that $\bar{\nu}=\mu \times \mu$. But that's impossible. To see this, apply Lemma 3 to $\bar{\nu}$. One learns that, for $i=1$ or 2 , among the integers $k$ with $T^{k} x \in D_{j}(i, 1 / 100)$, a fraction smaller than $2 \varepsilon$ of the $k$ also have $T^{k} y_{1} \in D_{j}(i, 1 / 100)$ and $T^{k} y_{2} \in D_{j}(i, 1 / 100)$, where $\varepsilon$ is arbitrary and $j$ depends on $\varepsilon$. Setting

$$
C_{j}=\left(D_{j}(1,1 / 100) \times D_{j}(2,1 / 100)\right) \cup\left(D_{j}(2,1 / 100) \times D_{j}(1,1 / 100)\right),
$$

we discover that $\bar{\nu}\left(C_{j}\right)<4 \varepsilon$, if $j$ is chosen sufficiently large. Yet $(\mu \times \mu)\left(C_{j}\right)>$ $\gamma^{2}$ for all $j$, where $\gamma$ is the lower bound on the measure of any tower that was mentioned in the first paragraph following the statement of the theorem. This rules out product measure for $\bar{\nu}$.

Hence $y_{1}$ and $y_{2}$ must be on the same orbit. Choose $k$ so that $y_{2}=T^{k} y_{1}$. Assume that $k \neq 0$ to obtain a contradiction. The assumption that both $\left(x, y_{1}\right)$ and $\left(x, y_{2}\right)$ are generic for $\bar{\mu}$ implies that $\bar{\mu}$ is $I \times T^{k}$-invariant.

Fix $A \subset X$ and define $\overline{\mathscr{B}}=\{\phi, X\} \times \mathscr{B}$, a $\sigma$-algebra on $X \times X$. Set $f(x)=E_{\bar{\mu}}(A \times X \mid \overline{\mathscr{B}})(x)$. For any $B \subset X$,

$$
\int_{B} f(x) d \mu=\bar{\mu}(A \times B)=\bar{\mu}\left(A \times T^{-k} B\right)=\int_{T^{-k_{B}}} f(x) d \mu=\int_{B} f\left(T^{k} x\right) d \mu .
$$

Since $B$ is arbitrary, $f(x)=f\left(T^{k} x\right)$, almost everywhere. Since $T$ is mixing, $T^{k}$ is ergodic, so $f$ is a constant almost everywhere. This makes $\bar{\mu}(A \times B)=$ $c \mu B$, where $c$ is a constant depending on $A$. That $\bar{\mu}$ has marginals $\mu$ implies that $c=\mu A$ and thus, $\bar{\mu}=\mu \times \mu$, which is a contradiction.

Hence $k=0, \bar{\nu}$ is diagonal measure, $y_{1}=y_{2}$, and the lemma is proved.

Lemma 5. if $\bar{\mu}$ is a self-joining of $T$ that is not product or off-diagonal, then there is a measure-preserving transformation $S$ of $X$, commuting with $T$, such that $S^{2}=T$ or $S^{2}=I$.

Proof. Suppose $(x, y)$ is generic for $\bar{\mu}$. Cut down to a subsequence of towers so that $x$ and $y$ are both always in $D_{j}(1 / 100)$. Lemma (2) tells us that $x$ and $y$ are on opposite $j$-towers for all sufficiently large $j$. 
For almost every $x$, there is a $y$ with $(x, y)$ generic for $\bar{\mu}$. Lemma 4 tells us that such a $y$ is unique. Let $S(x)=y$.

Because the set of points that are generic for $\bar{\mu}$ is product measurable, $S$ is measurable. The fact that $\bar{\mu}$ projects to $\mu$ along its second component implies that

$$
\mu A=\lim _{n \rightarrow \infty} \frac{1}{n} \sum_{i=0}^{n-1} \chi_{A}\left(T^{i}(S x)\right)=\lim _{n \rightarrow \infty} \frac{1}{n} \sum_{i=0}^{n-1} \chi_{S^{-1} A}\left(T^{i} x\right)=\mu S^{-1} A,
$$

and so $S$ is measure-preserving. Also, $(x, y)$ is generic for $\bar{\mu}$ exactly when $(T x, T y)$ is, from which we infer that $S$ commutes with $T$.

Now consider the joining $\bar{\nu}$ defined on rectangles by $\bar{\nu}\left(\begin{array}{lll}A & \times\end{array}\right)=$ $\mu\left(A \cap S^{-2} B\right) . \quad\left(x, S^{2} x\right)$ is generic for $\bar{\nu}$ for almost every $x \in X$. Were $x$ and $S^{2} x$ on different orbits of $T$, Lemma (2) would tell us that $\bar{\nu}$ is product measure, which is clearly impossible. Hence $S^{2} x=T^{k} x$ for some integer $k$ which apparently may depend on $x$. But the set $G_{k}=\left\{x \mid S^{2} x=T^{k} x\right\}$ is $T$-invariant and $\bigcup_{k=-\infty}^{\infty} G_{k}=X$. The ergodicity of $T$ implies that there is a particular $k$ with $\mu G_{k}=1$. In other words, $S^{2}=T^{k}$ almost everywhere. Replacing $S$ by $S \circ T^{\lfloor k / 2\rfloor}$, we get $S^{2}=T$ or $S^{2}=I$ (depending on the parity of $k$ ) and the lemma is proved.

We now state the few remaining words that are necessary to complete the proof of the theorem.

Proof of the theorem. If $\bar{\mu}$ is an ergodic self-joining of $T$ that is not product or off-diagonal measure, then Lemma (5) guarantees the existence of a measure preserving transformation $S$ that commutes with $T$ and such that either $S^{2}=$ $T$ or $S^{2}=I$. In the first case, the sets $B_{j}(1)$ can be seen to be a sequence of bases of towers of ever-increasing heights $\left(2 h_{j}(1)\right)$ and fuller measures, the levels of which generate the full $\sigma$-algebra $\mathscr{B}$. Hence $S$ is rank one. In the second case, we get a factor sub- $\sigma$-algebra by identifying the two point orbits of $S$. The factor measure-preserving transformation has for towers the image of $M_{j}(1)$ (or $\left.M_{j}(2)\right)$ under this identification. As $j$ increases, this gives us a generating sequence of towers for the factor, implying it is rank one. $T$ is seen to be a two point extension of this rank one factor.

\section{REFERENCES}

1. J. Baxter, A class of ergodic transformations having simple spectrum, Proc. Amer. Math. Soc. 27 (1971), 275-279.

2. J. King, For mixing transformations $\operatorname{rank}\left(T^{k}\right)=k \cdot \operatorname{rank}(T)$, Israel J. Math. $56(1986)$, 102-122.

3. D. S. Ornstein, On the root problem in ergodic theory, Proc. of the Sixth Berkeley Symposium on Mathematical Statistics and Probability, University of California Press, Berkeley, 1972, pp. 345-356.

4. D. Rudolph, An example of a measure-preserving map with minimal self-joinings, and applications, J. Analyse Math. 35 (1979), 97-122.

Department of Mathematics, The George Washington University, Washington, DisTRICT OF COLUMBIA 20052 